\title{
Atitudes de adultos com deficiência física frente ao idoso, à velhice pessoal e a pessoas com deficiência física
}

\author{
Physically disabled adults's attitudes toward elderly people, \\ personal old age, and other physically disabled persons
}

\author{
Marineia Crosara de RESENDE \\ Anita Liberalesso NERI ${ }^{2}$
}

\begin{abstract}
Resumo
O objetivo foi analisar atitudes em relação ao individuo portador de deficiência física, ao idoso e à própria velhice em 242 adultos de 24 a 39 anos, portadores de deficiência física congênita (6,4\%) ou adquirida. Apenas 4,5\% eram dependentes para AVDs, conforme o Barthel Index; 36,0\% usavam auxílios ortopédicos. Sendo 65,3\% mulheres; 47,0\%, casados, 53,3\% tinham oito anos de escolaridade, 35,0\%, 9 a 11 e 7,0\%, mais de 11. Foram submetidos a quatro escalas diferenciais Semânticas com 30 itens e quatro fatores cada (agência, cognição, relações sociais e persona). A análise de dados evidenciou que eles avaliaram mais positivamente o conceito de adulto portador de deficiência física do que o de idoso. Quanto mais antiga a convivência com a deficiência, mais positivas as atitudes em relação à velhice pessoal. Os dados são sugestivos do papel desempenhado pelos processos de autoregulação do self no manejo das conseqüências da incapacidade física sobre a vida pessoal e social.
\end{abstract}

Palavras-chave: velhice; idosos; deficiente físico; atitudes do adulto frente ao idoso.

\begin{abstract}
The aim was analyzing attitudes of physically disabled adults aged 24 to 39 toward physically disabled people, old people and own aging. There were 242 participants (6.4\% with congenital and $92.4 \%$ with acquired physical disability). Data collection involved application of the Barthel Index (4.5\% were dependent to ADLs; 36.0\% used orthopedic aids); a questionnaire assessing gender (65.3\% women), conjugal status (47.5\% married), schooling (53.3\% had eight years, $35.0 \% 9$ to $11,7.0 \%$ more than 11$)$, and four 30 items /four factors DS scales (agency, cognition, social relationships and persona). Data analysis showed that they evaluated more positively the concept of physically disabled people than the concept of old people; those that were congenitally disabled or had been disabled along the last 10 years or more scored higher on perspective of personal aging than those that had recently acquired disability. Data are suggestive of the role played by self regulatory processes in managing the consequences of disability on personal and social life.
\end{abstract}

Key words: old age; aged; physically disabled; adult attitudes.

A deficiência pode ser socialmente definida como o produto do descompasso entre as condições do indivíduo afetado por uma limitação funcional, as suas expectativas quanto à execução das atividades básicas e instrumentais de vida diária, as demandas ambientais nessa direção e a escassez ou a inadequação

\section{$\boldsymbol{\nabla} \boldsymbol{\nabla} \boldsymbol{v}$}

' Doutoranda em Educação, Faculdade de Educação, Universidade Estadual de Campinas; Docente, Centro Universitário do Triângulo, Uberlândia, MG, Brasil. E-mail:<marineiaresende@terra.com.br>.

Departamento de Psicologia Educacional, Faculdade de Educação, Universidade Estadual de Campinas. Rua Bertrand Russel, 801, 13083-970, Campinas, SP, Brasil. Correspondência para/Correspondence to: A.L. NERI. E-mail:<anitalbn@lexxa.com.br>. 
de condições instrumentais e sociais que the permitam funcionar adequadamente, mantendo a autonomia e a auto-estima. Além de recursos instrumentais, as pessoas com deficiência precisam de recursos pessoais para enfrentar os preconceitos e estereótipos existentes na sociedade, pois geralmente são objeto de discriminação e de preconceito, já que o ser diferente significa sobressair de forma negativa e assim gerar tensões e preconceitos (Montanari, 1999).

Geralmente não é esperado que crianças com deficiência se dêem bem na vida, mas há um número surpreendente de pessoas que, graças a uma combinação peculiar de condições ambientais e de características pessoais, apesar das limitações impostas pela deficiência, são bem-sucedidas na idade adulta. Rowe e Kahn (1998) citam o exemplo de Franklin Roosevelt, que, independentemente de conviver com as seqüelas da poliomielite, foi um dos melhores presidentes dos Estados Unidos. Stephen Hawking, considerado um dos maiores físicos do século XX, desenvolveu esclerose amiotrópica lateral, mas disse que, apesar de depender de uma cadeira de rodas e de outros aparatos tecnológicos, sua mente é livre para explorar os limites do universo.

As barreiras arquitetônicas e os preconceitos sociais manifestos em práticas sociais discriminativas e a ausência de políticas igualitárias podem agravar ainda mais as condições das pessoas que têm alguma deficiência física (Amaral, 1992; Silva, 1999), da mesma forma que afetam negativamente a vida das pessoas mais velhas. As dificuldades sociais enfrentadas pelas pessoas com deficiência física e pelas pessoas idosas principalmente doentes ou fisicamente incapacitadas não raro são causadas pelas deficiências da sociedade em atender e aceitar suas necessidades específicas (Araújo, 1997). Uma das grandes lutas das pessoas com deficiência física é poderem ser diferentes sem estarem em desvantagem. Segundo Berger (1999), grande parte das pessoas que não têm deficiência física possui atitudes discriminatórias que parecem decorrer da dificuldade de conviver com a diferença, mesmo as que de fato podem vir afetar a qualquer um por serem decorrentes de acidente ou doença inesperada (ver também, Katz, Hass e Bailey (1988), Lys e Pernice (1995) Georges (1997), Beattie, Anderson e Antonak 124 (1997)).
Para Grayson e Marini (1996) e Maras e Brown (1996), as atitudes negativas, as ambivalências e a distância social percebida são minimizadas quando existe contato mais próximo com pessoas com deficiência e quando existe conhecimento de suas expectativas frente à vida. As atitudes das pessoas com deficiência estão intimamente ligadas à percepção sobre sua qualidade de vida, entendida como noção geral de bem-estar. Em pesquisa com 153 pessoas com deficiência, 54,3\% dos participantes responderam ter excelente ou ótima qualidade de vida e que, portanto, tinham uma atitude positiva frente à deficiência física (Albrecht \& Devlieger, 1999).

Ao contrário do que ditam os preconceitos, velhice não é sinônimo de incapacidade funcional e de dependência. Estudos epidemiológicos realizados em vários países apontaram que apenas $4 \%$ dos idosos com 65 anos ou mais apresentam incapacidade grave e alto grau de dependência (Gatz, 1995). No Brasil, os dados do Censo Demográfico de 1991 mostraram que, embora entre os idosos haja uma maior proporção de pessoas com deficiência do que entre o restante da população, essa proporção não ultrapassa 4\% (Camarano \& Ghaouri, 1999). A deficiência física resulta principalmente de doenças crônicas e tem prevalência maior entre as mulheres do que entre os homens idosos. Se há um aumento no número de doenças, há também um aumento no risco de diminuição na capacidade para execução das atividades básicas e instrumentais de vida diária, bem como há diminuição na mobilidade (Fried, Bandeen-Roche, Kasper \& Gubralnik, 1999), o que acarreta aumento da demanda por ajuda instrumental.

A presença de algum grau de incapacidade para o desempenho de atividades básicas e instrumentais de vida diária não significa necessariamente impedimento para a continuidade do funcionamento cognitivo e emocional; o que significa que as pessoas adultas e idosas, portanto podem ser fisicamente incapazes e dependentes, mas podem preservar a autonomia, manifesta na capacidade de tomar decisões. Quando mantêm a integridade cognitiva e a integridade dos mecanismos de auto-regulação do self, adultos e idosos com deficiência física podem ativar mecanismos de adaptação para enfrentar perdas em funcionalidade. Podem recorrer à procura de recursos tecnológicos, buscar apoio social e psicológico ou exercer controle secundário sobre o comportamento 
de outras pessoas para compensar suas limitações (Neri, 2000). Entretanto, existe forte relação entre deficiência física, limitações funcionais e baixos níveis de recursos psicológicos (Kempen, Heuvelen, Sonderer, Brink, Kooijman \& Ormel, 1999). Em pesquisa realizada com 624 idosos, foi relatado que as limitações funcionais afetaram o desempenho das atividades básicas e instrumentais de vida diária, com fortes repercussões sobre o funcionamento psicossocial dos idosos.

Na velhice, a probabilidade de ocorrência de perdas físicas, psicológicas e sociais é maior do que a ocorrência de vantagens evolutivas, porque o envelhecimento normal prevê um processo de contração. No entanto, mesmo na presença de perdas que podem ser reduzidas ou ampliadas, dependendo das condições da pessoa -, o ser humano sempre aspirou a viver longamente e com saúde. A pesquisa gerontológica tem apontado que isso é possível e que, de fato, uma velhice bem-sucedida está associada à boa saúde física e mental, atividade e envolvimento com a vida, condições essas dependentes da influência combinada de eventos genético-biológicos, sociais e psicológicos (Rowe \& Kahn, 1997). Ou seja, a atitude frente à velhice é influenciada pelas crenças que a pessoa detém em sua habilidade para ativar motivação, resgatar cognição e mecanismos de ação, que são necessários para exercer controle sobre tarefas específicas.

As atitudes e as crenças que o indivíduo detém sobre si mesmo e sobre suas relações com o mundo são um aspecto essencial da adaptação do self. Elas são formadas a partir da experiência direta e da interação social e desempenham papel central nos esforços de ajustamento pessoal. Graças ao concurso de mecanismos de auto-regulação do self, atuando com diferentes graus de eficácia, adultos e idosos em diferentes graus de funcionalidade física podem lidar com as perdas do processo de envelhecimento e também adaptar-se a eventuais desvantagens e incapacidades ocorridas na velhice ou em fases anteriores do desenvolvimento. Em qualquer dos casos, ocorre mobilização em direção ao ajustamento de suas crenças, avaliações e expectativas em relação a si mesmo, em comparação a outras pessoas que vivem experiências evolutivas semelhantes às suas (Bandura, 1986; 1997).
Mecanismos de comparação social intervêm na maneira como as pessoas no geral e, de modo especial, os adultos com deficiência física antecipam e vivem a própria velhice. De certa forma, a sua condição é análoga a de idosos que apresentam incapacidade física, dado que necessitam de ajuda tecnológica e de auxílio instrumental de outras pessoas, além de sofrerem os efeitos de preconceitos e de limitações em oportunidades. No entanto, além disso, é provável que antevejam que as suas dificuldades físicas e sociais atuais podem ser potencializadas pelo envelhecimento. Semelhante condição é possivelmente importante no estabelecimento e na manutenção de crenças sobre o próprio funcionamento e desenvolvimento pessoal, motivo pelo qual é importante que tais crenças sejam conhecidas pelos profissionais que podem providenciar ajuda a essas pessoas.

A despeito das precariedades notadas no atendimento social às pessoas com deficiências, a melhora nas condições gerais de saúde pública - um dos fatores que está na base do processo de envelhecimento populacional, está ensejando um aumento no número de pessoas que envelhecem, ainda que apresentem uma deficiência física congênita ou anteriormente adquirida. Acreditamos que se conhecermos o que elas pensam acerca da velhice e do seu próprio envelhecer poderemos compreender melhor aspectos singulares do seu envelhecimento, bem como poderemos contribuir para a sua preparação para um envelhecimento saudável, com melhor qualidade de vida. Pesquisar suas atitudes em relação à velhice e à deficiência física pode oferecer subsídios para a compreensão dos mecanismos de autoregulação da personalidade que intervém no bem-estar subjetivo desses indivíduos e pode oferecer subsídios para as práticas clínica e social. Com base nesse raciocínio, planejamos uma pesquisa de levantamento para responder às seguintes questões: Quais são as atitudes das pessoas com deficiência física em relação aos idosos? Quais as expectativas de velhice de indivíduos não idosos com deficiência física? Há relações entre as atitudes em relação a essas duas categorias? Esse questionário tem como objetivo: 1) verificar a relação entre a atitude de pessoas com deficiência física e o idoso, a própria velhice e a pessoa 
com deficiência física; 2) averiguar a relação entre a atitude de pessoas com deficiência física e o idoso e a própria velhice; 3) reconhecer a relação entre as atitudes de pessoas com deficiência física e o idoso e a pessoa com deficiência física; 4) analisar a relação entre as atitudes de pessoas com deficiência física e a velhice pessoal e a pessoa com deficiência física.

\section{Método}

Participaram voluntariamente deste estudo, 242 pessoas com deficiência física, associadas da Associação dos Paraplégicos de Uberlândia (APARU). Os sujeitos eram alfabetizados, com idade entre 24 e 39 anos; na amostra de conveniência, 65,3\% eram mulheres, com idade média de 32,17 anos ( $D P=4,42$ anos). O número de casados era maior (47,5\%) do que o de solteiros (37,2\%), separados $(11,2)$ e viúvos (1,2\%) e 2,9\% não informaram estado civil. Tinham até oito anos de escolaridade 53\% dos sujeitos e 35,1\% entre nove e onze; 7,0\% fizeram curso superior e 0,8\% não responderam. Com relação à origem da deficiência, 6,4\% tinham deficiência física congênita e 92,4\% apresentaram deficiência física adquirida. Trinta e dois por cento conviviam com a deficiência física há mais de dez anos, sendo que 39,3\% deles desde os dois primeiros anos de vida e 51,2\% a partir da vida adulta (9,5\% não responderam). Dos sujeitos, 36,0\% utilizavam auxílio ortopédico (cadeira de rodas, muletas, bengalas e aparelhos ortopédicos). A maioria dos participantes $(85,5 \%)$ era totalmente independente para as atividades básicas de vida diária, ou seja, dispunha de compensações ou apoios tecnológicos para desempenhá-las. Apenas 4,5\% puderam ser considerados dependentes.

A pesquisa envolveu a utilização de quatro instrumentos que foram apresentados aos sujeitos num formulário impresso, numa única sessão de coleta de dados, em que se solicitavam respostas escritas:

a) Questionário informativo sobre os sujeitos composto por itens abertos, fechados e mistos que solicitavam informações sobre dados pessoais (idade, estado civil, renda familiar, ocupação, se trabalha ou é aposentado) e dados sobre a deficiência (nome da deficiência, se congênita ou adquirida, idade em que adquiriu a deficiência e se utiliza auxílio ortopédico).

b) Escala para avaliação de atitudes em relação ao idoso e à velhice pessoal (Neri, 1991; 1997) - utilizada para avaliar atitudes em relação à velhice, é composta por 30 escalas de cinco pontos ancorados por pares de adjetivos com significados opostos, pertencentes aos domínios cognição, agência, relacionamento social e persona. Com ela foram avaliados os conceitos "O idoso é" e"Quando eu for velho eu serei".

c) Escala "a pessoa com deficiência física é" composta pelos mesmos itens que avaliaram os conceitos idoso e velhice pessoal.

d) Índice Barthel para avaliação de independência no desempenho de atividades de vida diária - trata-se de instrumento para avaliar a capacidade funcional para o desempenho de atividades básicas de vida diária, permitindo, assim, a sobrevivência sem ajuda (Mahoney \& Barthel, 1965).

\section{Resultados}

Os dados foram submetidos a análises descritivas e a testes não-paramétricos univariados e bivariados. Houve predominância de respostas positivas e de respostas negativas fracas ou moderadas para todos os objetos estudados. Os resultados serão apresentados na seguinte seqüência: a) Atitudes dos sujeitos em relação ao idoso, à velhice pessoal e à pessoa com deficiência física; b) Relações entre as respostas aos conceitos "O idoso é" e "Quando eu ficar velho eu serei"; c) Relações entre as atitudes frente ao idoso e à pessoa com deficiência física; d) Relações entre as atitudes frente à velhice pessoal e à pessoa com deficiência física.

\section{Conceitos:}

"O idoso é": os sujeitos com deficiência física demonstraram atitudes mais positivas e neutras que atitudes negativas em relação ao velho. Atitudes positivas foram apresentadas principalmente por mulheres $(p=0,0040)$, por aqueles com menor escolaridade e por pessoas com deficiência física congênita, particularmente no domínio agência $(p=0,0346)$ (Figura 1). 


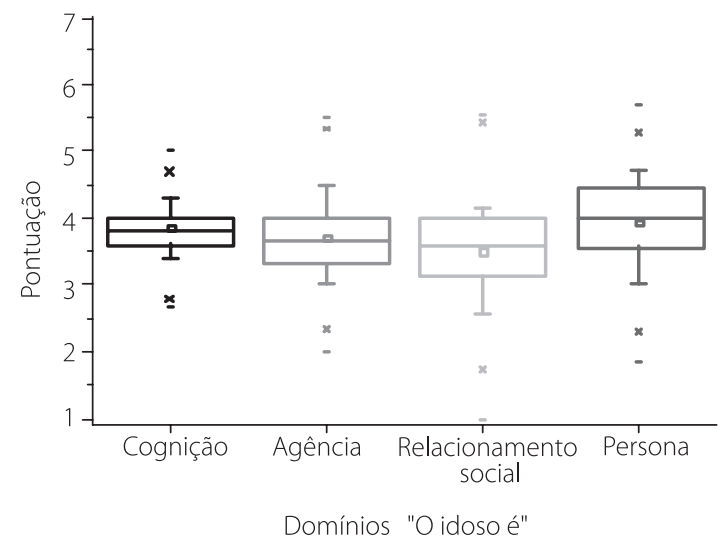

Figura 1. Distribuição dos valores, médias e variabilidade de valores obtidos para os domínios da escala "O idoso é".

Ao responderem ao instrumento apresentado, os sujeitos avaliaram que o idoso bem-sucedido é aquele que apresenta características de sabedoria, persistência, rapidez e criatividade; que se mantém ativo, esperançoso e produtivo; e que é generoso, construtivo, cordial, bem-humorado, agradável e interessado pelas pessoas. Envelhecer mal apareceu relacionado à imprecisão, embotamento, dependência, desconfiança, rejeição e desvalorização social. As pessoas com nível mais alto de escolaridade foram as que tiveram uma visão menos positiva dos velhos, provavelmente em função de ter um nível de aspiração mais alto, serem mais informadas e terem mais acesso às oportunidades e aos bens de consumo.

"Quando eu ficar velho eu serei": com relação ao próprio envelhecimento, as pessoas com deficiência física também relataram uma atitude positiva, sendo que as mulheres têm visão mais positiva que os homens, principalmente nos domínios: cognição $(p=0,0013)$, agência $(p=0,0136)$ e relacionamento social $(p=0,0299)$. As pessoas com deficiência física com maior índice de dependência para as AVDs $(p=0,0139)$ têm visão menos positiva em relação ao envelhecimento, nos domínios agência $(p=0,0221)$, relacionamento social $(p=0,0150)$ e persona ( $p=0,438)$ (Figura 2).

Em relação à própria velhice, os indivíduos com deficiência física apresentaram expectativas muito positivas, e mais positivas que em relação ao idoso atual. Esperam ser velhos sábios, persistentes, criativos, claros, concentrados, flexíveis, ativos, esperançosos, produtivos, saudáveis, independentes, generosos, interessados pelas

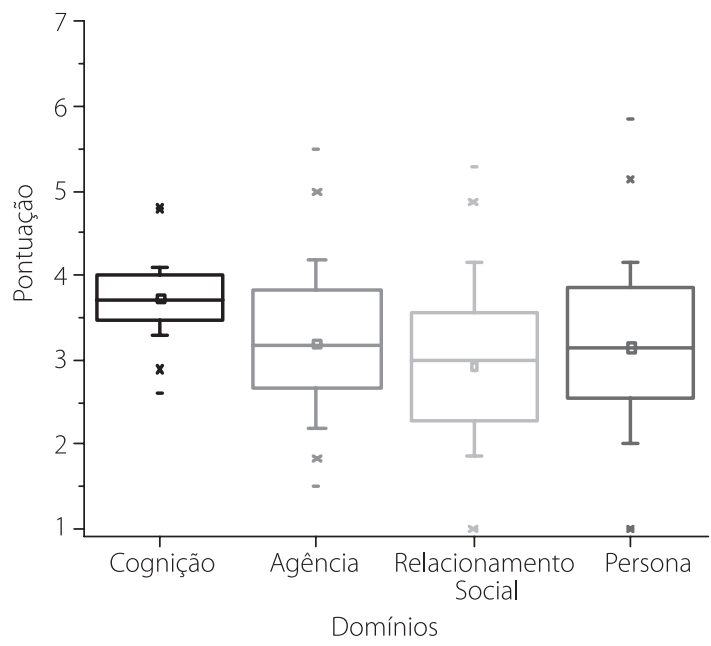

Figura 2. Distribuição dos valores, médias e variabilidade de valores obtidos para os domínios da escala "Quando eu ficar velho eu serei".

pessoas, construtivos, cordiais, bem-humorados, confiantes, agradáveis, progressistas, atualizados, valorizados, integrados e aceitos. No entanto, acreditam que terão também características negativas, pois serão velhos imprecisos, embotados, inseguros, lentos, deprimidos e rejeitados. Nesse sentido, não diferem de pessoas sem deficiência física.

"A pessoa com deficiência física é": a atitude das pessoas com deficiência física em relação a outras pessoas na mesma condição que elas também foi positiva, sendo que as mulheres $(p=0,0006)$ relataram atitudes mais positivas que os homens, especialmente para os domínios cognição $(p=0,0001)$ e relaciona-mento social ( $p=0,0050)$ (Figura 3).

As pessoas mais dependentes para as atividades de vida diária $(p=0,0030)$ apresentaram visão menos positiva em relação à pessoa com deficiência física, principalmente nos domínios agência $(p=0,0094)$ e relacionamento social $(p=0,0166)$. As pessoas que estavam separadas ou divorciadas $(p=0,0080)$ foram as que tiveram visão mais positiva da pessoa com deficiência física, seguidas pelos casados e, por último, pelos solteiros e viúvos. As pessoas que adquiriram deficiência física com trinta anos ou mais são as que relataram atitude mais positiva em relação às pessoas com deficiência física ( $p=0.0309)$, principalmente no domínio relacionamento social $(p=0,0337)$. As 


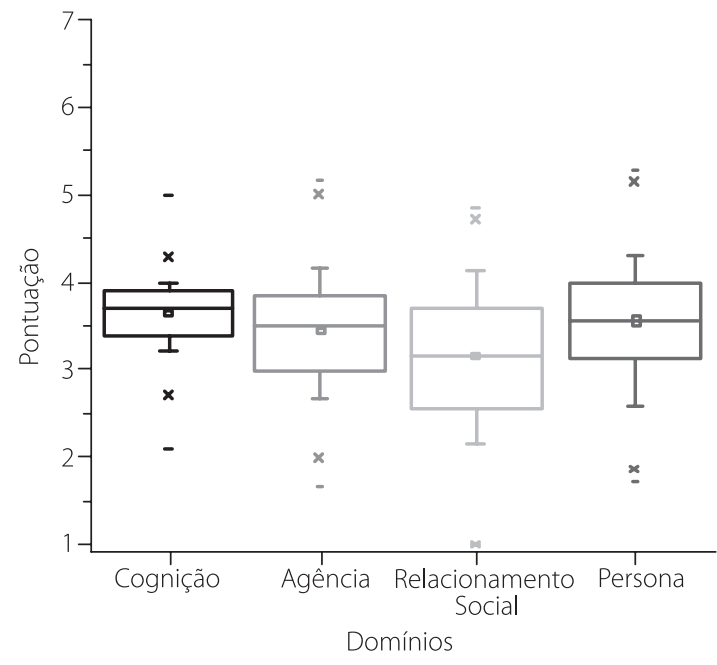

Figura 3. Distribuição dos valores, médias e variabilidade de valores obtidos para os domínios da escala "O portador de deficiência física é".

mulheres apresentaram expectativa de que terão uma velhice melhor que os homens, acham que quando envelhecerem estarão mais aptas para processar informações e solucionar problemas e, conseqüentemente, serão mais adaptadas em termos sociais, afetivos e emocionais. Elas também se projetaram como mais autônomas e capazes de realizar tarefas no futuro do que os idosos atuais. As mulheres têm conquistado cada vez mais espaço na sociedade, são maioria em programas oferecidos por centros de convivência e Universidades da Terceira Idade, o que aumenta o seu senso de controle e poder, seu senso de autonomia e sua crença que reúne qualidades para ter uma velhice bem-sucedida (Debert, 1999; Neri, 2000). A maior visibilidade que essas mulheres idosas têm hoje no Brasil provavelmente afetará as crenças dos mais jovens em relação a terem uma boa velhice.

As pessoas com deficiência física congênita se descreveram como autônomas e capazes de realização. Provavelmente essas pessoas estejam bem adaptadas, já que convivem com a deficiência há pelo menos 24 anos (idade mínima para participação no estudo). Possivelmente desenvolveram mecanismos de seleção, otimização e compensação do self (Baltes \& Baltes, 1990) que Ihes permitem melhor adaptação e Ihes permitem também vislumbrar a continuidade de suas condições atuais até a velhice. Tanto o sucesso no manejo da deficiência quanto o envelhecimento bem-sucedido dependem da seleção de domínios comportamentais em que o indivíduo detém melhor nível de funcionamento e da otimização desse funcionamento através de estratégias que compen-sem as perdas.

Ainda em relação ao próprio envelhecimento, as pessoas que apresentam maior dependência para as atividades de vida diária foram também as que apresentaram atitude menos positiva em relação à autonomia e à capacidade para realização de tarefas, o que demonstra que essas pessoas estão conscientes de sua condição em relação ao futuro, não negam a própria realidade, indicativos de adaptação que as pode favorecer. Se apresentam algum grau de dependência enquanto são jovens, sabem que continuarão dependendo, e que, em alguns casos sua dependência poderá aumentar quando chegarem à velhice. No entanto, é importante lembrar que embora a associação entre deficiência, dependência e velhice diminua as possibilidades de envolvimento social, mesmo que esses indivíduos não apresentem padrões ótimos em determinados domínios, eles poderão obtê-los em outros (Heckenhausen \& Schulz, 1996).

As pessoas solteiras ou viúvas apresentam uma atitude mais negativa frente à velhice do que as casadas e descasadas. Esse dado possivelmente reflita a evidência empírica de que os idosos casados têm melhor qualidade de vida do que os que vivem sozinhos, principalmente se são homens. Dados da pesquisa internacional confirmam que ser mulher, sozinha e idosa aumenta as chances de incapacidade e doenças, ao passo que ser casado é preditivo de melhor velhice. Ou seja, as pessoas casadas proporcionam mais cuidado do que as viúvas e solteiras, o que não necessariamente significa que recebam mais suporte social ou afetivo que os não casados. O que ocorre é que talvez pessoas casadas tenham mais competência para dar apoio e cuidar outro que os não casados (Wagner, Schütze \& Lang, 1999).

As atitudes das pessoas com deficiência física em relação a outras pessoas na mesma condição que elas também foram positivas, sendo que as mulheres relataram atitudes mais positivas que os homens, especialmente para os domínios cognição e relacionamento social. As pessoas mais dependentes 
para as atividades básicas de vida diária apresentaram visão menos positiva em relação à pessoa com deficiência física, principalmente nos domínios agência e relacionamento social. As pessoas que estavam separadas ou divorciadas foram as que tiveram visão mais positiva da pessoa com deficiência física, seguidas pelos casados e, por último, pelos solteiros e viúvos. As pessoas que adquiriram deficiência física com 30 anos ou mais são as que relatam atitude mais positiva em relação às pessoas com deficiência física, principalmente no domínio relacionamento social.

Os dados encontrados nesse item estão de acordo com o que as pessoas com deficiência física pensam sobre a velhice pessoal, sugerindo uma expectativa de continuidade no desenvolvimento. Os dados são sugestivos dos efeitos do conhecimento socialmente compartilhado sobre a natureza e a seqüência das mudanças evolutivas. Podemos inferir que as atitudes e as autocrenças sobre o envelhecimento, o velho e a pessoa com deficiência física agem como forças orientadoras para proteção do selfe como processos adaptativos mediados por comparações sociais nos nossos sujeitos.

"O velho é versus" quando eu ficar velho eu serei": os participantes têm uma visão diferente do "idoso é"e de "quando eu ficar velho eu serei", isto é, eles têm uma visão mais positiva sobre a própria velhice do que sobre o velho. Eles têm uma leve tendência a ter atitude negativa em relação ao idoso, um pouco mais negativa do que a que apresentaram em relação à pessoa com deficiência física (Figura 4).

"A Pessoa com Deficiência Física é" versus "Quando Eu Ficar Velho Eu Serei": aqueles com atitude positiva em relação à pessoa com deficiência física tendem a ter também uma atitude positiva em relação à própria velhice (Figura 5).

Os respondentes que acreditam ser possível viver bem apesar da deficiência na juventude acreditam que terão uma velhice bem-sucedida, que é possível ser feliz na velhice e continuar realizando seus sonhos e aspirações, com uma imagem positiva do velho e do próprio envelhecimento.

Os resultados observados corroboram evidências da pesquisa internacional segundo a qual as

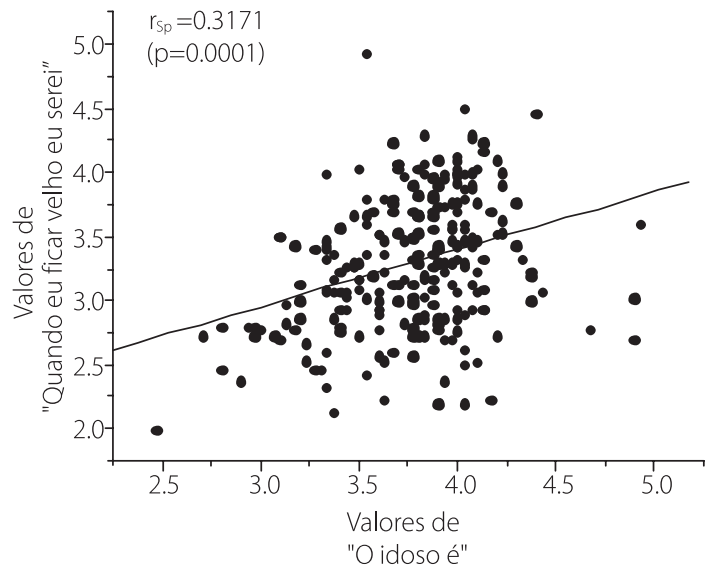

Figura 4. Valores das correlações entre as respostas às escalas "O velho é" e "Quando eu ficar velho eu serei".

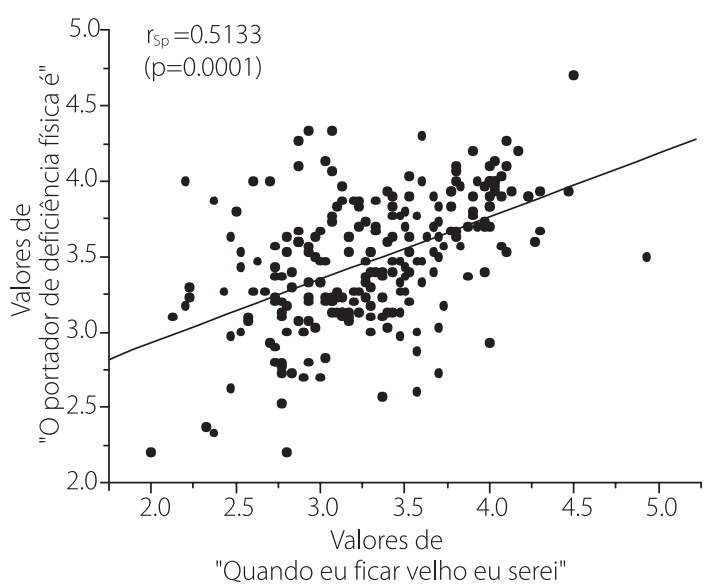

Figura 5. Valores das correlações entre as respostas às escalas "Quando eu ficar velho eu serei" e "A pessoa com deficiência física é".

concepções dos adultos sobre o desenvolvimento são comparáveis às normas e expectativas de desem-penho apropriado às diferentes idades encontradas na sociedade. Expectativas e normas amplamente compartilhadas fornecem um sistema de referência para as pessoas em relação ao qual avaliarão as próprias trajetórias evolutivas (Heckhausen, Dixon \& Baltes, 1898; Heckhausen, 1999). Nessas avaliações, processos de comparação social com padrões avaliados como inferiores, iguais ou superiores às próprias competências e características desempenham papel central na manutenção da auto-estima e nas crenças de eficácia pessoal e realização (Wood \& Taylor, 1991; Heckhausen, 1999). 
A atitude que as pessoas com deficiência física apresentam em relação ao próprio envelhecimento provavelmente é influenciada pelas crenças e atitudes que elas apresentam frente à deficiência. Por isso, conhecer suas atitudes frente à deficiência ajuda a compreender como elas imaginam o futuro e como elas esperam lidar com a velhice, o que pode dar indícios aos profissionais de como estruturar programas educativos voltados a pensar o processo de envelhecimento, particularmente das pessoas com deficiência física.

A deficiência é apenas mais uma característica de quem a tem. Apesar de exercer influência sobre os indivíduos, outros aspectos relativos à personalidade são preponderantes na sua forma de encarar o mundo. O convívio social e as regras do grupo têm influência sobre a construção e a manutenção das crenças e atitudes e, assim, os indivíduos de uma mesma coorte tendem a apresentar atitudes semelhantes frente aos mesmos objetos de julgamento e avaliação.

As crenças de auto-eficácia desempenham um papel importante nas relações dos indivíduos com o mundo porque refletem as suas avaliações quanto à própria capacidade de se comportar de forma adequada e produtiva em situações específicas. Influenciam os tipos de atividades que as pessoas escolherão, o esforço que vão imprimir em suas ações, sua perseverança frente às dificuldades, suas auto-avaliações, suas avaliações sobre o mundo externo e suas experiências emocionais (Bandura, 1986). É especialmente interessante notar que no caso dos nossos sujeitos, acreditar que é possível ser eficaz na velhice, mesmo na presença de incapacidades físicas, é um preditor importante de envolvimento social, auto-estima preservada e bem-estar subjetivo. Essa crença encontra fundamentos em literatura psicogerontológica, que encontrou relações entre crenças de auto-eficácia e preservação do estilo e da qualidade de vida em idosos mesmo na presença de incapacidades físicas (Seeman, Unger, McAvay \& Icon, 1999).

O alcance dessas competências exige a combinação de diversos fatores biológicos, psicológicos e sociais e depende das oportunidades que o indivíduo teve ao longo da vida e de condições adequadas para sua sobrevivência e desenvolvimento, tais como educação, saúde, habitação e trabalho.

\section{Conclusão}

A visão do envelhecimento e da deficiência começam, a passos tímidos, a tomar novos rumos. 0 que uma pessoa observa, acredita e vive está fortemente relacionado ao seu contexto social e à sua história de vida. O que as pessoas almejam é viver bem, com qualidade de vida, independentemente de sua idade, tendo ou não uma deficiência física. A convivência com pessoas idosas e pessoas com deficiência têm dado oportunidade à quebra de preconceitos e tabus.

O estudo multidisciplinar de envelhecer com uma deficiência é uma área nova e emergente, tanto no campo da Gerontologia como no das Ciências Sociais e da Saúde. Nosso estudo é pioneiro no Brasil e muito ainda precisa ser investigado sobre a relação entre deficiência e envelhecimento.

\section{Referências}

Albrecht, G.I., \& Devlieger, P.J. (199(). The disbility paradox: high quality of life against all odds. Social Science and Medicine, 48 (8), 977-988.

Amaral, L.A. (1992). Espelho convexo: o corpo desviante no imaginário coletivo, pela voz da literatura infanto-juvenil. São Paulo:SP. Tese de doutorado em Psicologia, Instituto de Psicologia, Universidade de São Paulo.

Araújo, L.A.D. (1997). A proteção constitucional das pessoas portadoras de deficiência (2a ed.). Brasília, CORDE.

Baltes, P.B., \& Baltes, M.M. (1990). Psychological perspectives on successful aging: the model of selective optimization with compensation. In P.B. Baltes, \& M.M. Baltes (Eds.) Successful Aging: perspectives from the behavioral sciences. Cambridge: University Press.

Bandura, A. (1986). Social foundations of thought and action: a social cognitive theory (Prentice-Hall series in social learning theory). New Jersey: Prentice-Hall.

Bandura, A. (1997). Self-efficacy: the exercise of control. New York: W.H. Freeman and Company.

Beattie, J.R., Anderson, R.J., \& Antonak, R.F. (1997). Modifying attitudes of prospective educators toward students with disabilities and their integration into classrooms. The Journal of Psychology, 131 (3), 245-259.

Berger, M. (1999). A Projeção da Deficiência. Dissertação de mestrado em Antropologia Social, Faculdade de Filosofia, Letras e Ciências Humanas, Universidade de São Paulo.

Camarano, A.A., \& Ghaouri, S.K.E. (1999). Idosos brasileiros: que dependência é essa? In A.A. Camarano, (Org.). Muito além dos 60: os novos brasileiros. Rio de Janeiro: IPEA. 
Debert, G.G. (1999). A reinvenção da velhice: socialização e processos de reprivatização do envelhecimento. São Paulo: EDUSP.

Fried, L.P, Bandeen-Roche, K., Kasper, J.D., \& Gulralnik, J.M. (1999). Association of comorbidity with disability in older woman: the woman's health and aging study. Journal of Clinical Epidemiology, 52 (1), 27-37.

Gatz, M. (1995). Questions that aging puts to preventionities. In L.A. Bond, S.J. Cutler, \& A. Grans (Eds.). Promoting successful and productive aging. Thousand Oaks, California: SAGE.

Georges, C.A. (1997). Youngster has HIV, poor attention span; Is he really disabled? The All Street Journal C, (65), October 1.

Grayson, E., \& Marini, I. (1996). Simulated disability exercises and their impact on attitudes toward persons with disability. International Journal of Rehabilitation Research, $19(2), 123-131$.

Heckhausen, J., Dixon, R.A., \& Baltes, P.B. (1989). Gains and losses in development throughout adulthood as perceived by different adult age groups. Developmental Psychology, 25, 109-121.

Heckhausen, J. (1999). Developmental regulation in adulthood: age-normative and sociostructural constraints as adaptative challenges. Cambridge: Cambridge University Press.

Katz, I., Hass, R.G., \& Bailey, J. (1988). Attitudinal ambivalence and behavior toward people with disabilities. In H.E. Yuker (Ed.). Attitudes Toward Persons With Disabilities. New York: Springer.

Kempen, G.I.J.M., Heuvelen, M.J.G., Sonderer, E., Brink, R.H.S., Kooijman, A.C., \& Ormel, J. (1999). The relashionship of functional limitations to disability and the moderating effects of psychological attributes in communitydwelling older persons. Social Science and Medicine, 48 (9), 1161-1172.

Lys, K., \& Pernice, R. (1995). Perceptions of positive attitudes toward people with spinal cord injury. International Journal of Rehabilitation Research, 18, 35-43.

Mahoney, F.L., \& Barthel, D.W. (1965). Functional evaluation: the Barthel Index. State Medical Journal (4), 61-65.
Maras, P., \& Brown, R. (1996). Effects of contact on children's attitudes toward disability: a longitudinal study. Journal of Applied Social Psychology, 26 (23), 2113-2134.

Montanari, P.M. (1999). Jovens e deficiência: comportamento e corpos desviantes. In N. Schor, M.S.F.T. Mota, \& V.C. Branco (Orgs.). Cadernos juventude, saúde, desenvolvimento (pp.97-108). Brasília: Ministério da Saúde, Secretaria de Políticas de Saúde.

Neri, A.L. (1991). Envelhecer num país dejovens: significados de velho e velhice segundo brasileiros não idosos. Campinas: Unicamp.

Neri, A.L. (1997). Atitudes em relação à velhice: evidências de pesquisa no Brasil. Gerontologia, 5 (3), 130-139.

Neri, A.L. (2000). Envelhecimento e qualidade de vida na mulher. Texto não publicado, circulação restrita ao Programa de Pós-Graduação em Gerontologia da Universidade Estadual de Campinas.

Rowe, J.W., \& Kahn, R.L. (1997). Successful aging. Gerontologist, 37 (4), 433-440.

Schulz, R., \& Heckhausen, J. (1996). A life span model of successful aging. American Psychologist, 51 (7), 702-714.

Seeman, T.E., Unger, J.B., McAvay, G., \& Icon, C.F.M. (1999). Self-efficacy beliefs and perceived declines in functional ability: MacArthur studies of successful aging. Journal of Gerontology; Psychological Sciences, 54B, (4), 214-222

Silva, A.M.F. (1999). Refletindo sobre a qualidade de vida do portador de deficiência: resgatando os direitos do cidadão. Texto Contexto Enfermagem 8, (3), 88-98.

Wagner, M., Schütze, Y., \& Lang, F.R. (1999). Social Relationships in Old Age. In P.B. Baltes, \& K.U. Mayer (Ed.). The Berlin Aging Study: Aging from 70 to 100. New York: Cambridge University Press.

Wood, J.V., \& Taylor, K.L. (1991). Serving self relevant goals through social comparison. In J.M. Suls, \& T.A. Willis (Eds.). Social comparison: contemporary theory and research. Hillsdale, NJ: Erbaum.

Recebido para publicação em 18 de julho de 2003 e aceito em 17 de março de 2005. 
